\title{
Beam Steering and Dynamic Focusing by Coherent Control of Light with Light on Metasurfaces
}

\author{
Fei He${ }^{1}$, Kevin F MacDonald ${ }^{2} \&$ Xu Fang ${ }^{1}$ \\ ${ }^{I}$ School of Electronics and Computer Science, University of Southampton SO17 1BJ, UK \\ ${ }^{2}$ Optoelectronics Research Centre and Centre for Photonic Metamaterials, University of Southampton SO17 1BJ, UK \\ x.fang@soton.ac.uk
}

\begin{abstract}
We report continuous 'coherently controlled' tuning of the wavefront of light scattered by phase-gradient silicon metasurfaces, which enables optical-frequency beam steering and dynamic focusing without moving parts. (C) 2020 The Authors
\end{abstract}

\section{Introduction}

In recent years, it has been a focus of the metasurface research field to achieve active tuning of optical metasurfaces. For many applications, e.g. light detection and ranging (lidar) systems and augmented reality, it is of particular importance that the tuning results in a continuous range rather than a discrete set of functional output states. A widely explored approach to achieve dynamic, continuous tuning of metasurfaces is to manufacture them from materials which themselves have intrinsically tuneable properties, such as $\mathrm{VO}_{2}$ and GST. Another approach relies on reversible structural deformation of metasurfaces, which necessitates the use of soft, easily deformable materials as the substrate or host matrix. Both of these approaches depend on physical changes to the metasurface, either to the constituent materials or to the morphology of the whole array. Here, we introduce a new approach that facilitates continuous beam steering and dynamic focusing by using coherent control of metamaterial response; i.e. without changes to the intrinsic material properties and the morphology of the metasurface.

In the context of photonic metamaterials, coherent control/illumination refers to the use of two coherent, counter-propagating light beams to control the electromagnetic responses of metasurfaces or ultra-thin metamaterials [1]. It makes use of the fact that an ultrathin (subwavelength thickness) metasurface responds only to the local electromagnetic field in the metamaterial plane. If the metasurface is positioned at the electric antinode, which is also the magnetic node, of a standing wave formed in the coherent illumination, its electric responses are enhanced while its magnetic responses are suppressed. The same metasurface positioned at the magnetic antinode, which is also the electric node, of the standing wave, reveals its magnetic characteristics instead.

In this work, we explore the continuous, intermediate field configurations between the two extremes of field nodes and antinodes, and apply them on silicon-based phase gradient metasurfaces. We demonstrate via numerical simulation that the phase gradient, which determines the wavefront of scattered light based on the generalised Snell's law [2], can be tuned via coherent illumination. In a beam-steering metasurface, the output beam can be steered/deflected continuously over an angular range of $\sim 10^{\circ}$. In a metalens, the focal length varies dynamically by up to $18 \%(\sim 20 \mu \mathrm{m})$.

\section{Metasurface and illumination configurations}
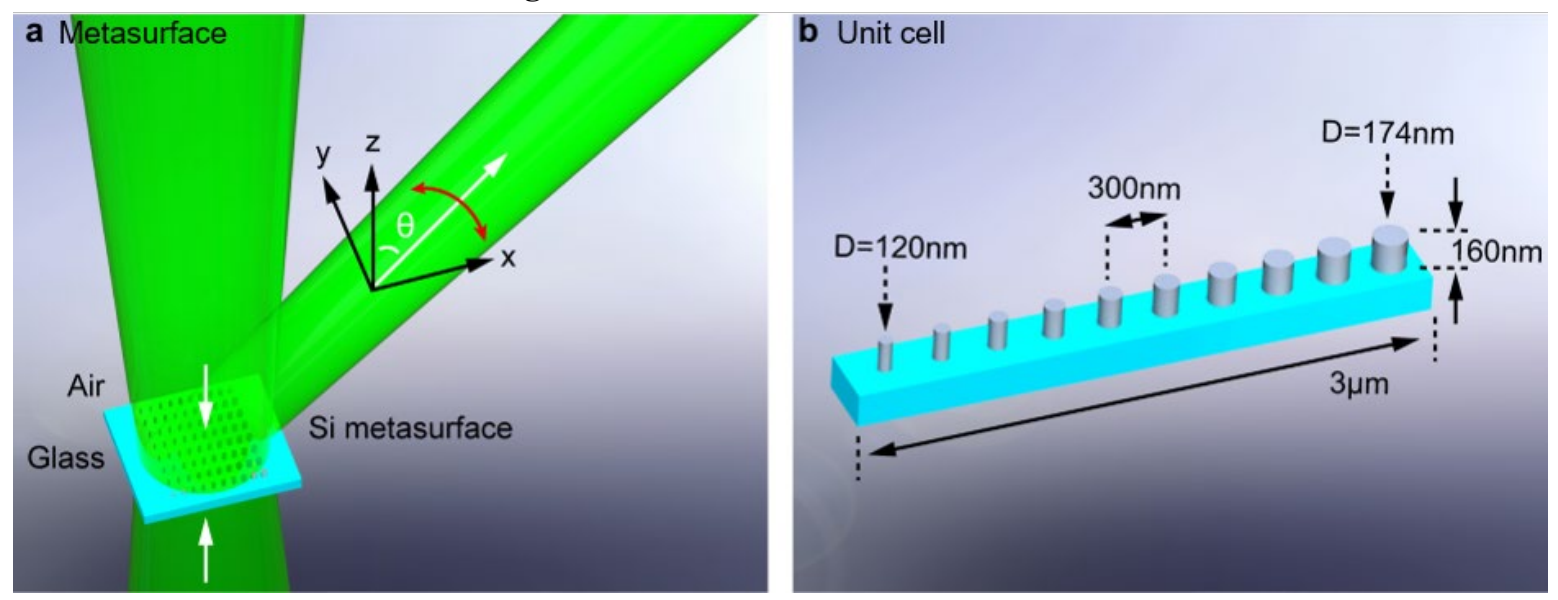
Fig. 1. Schematic diagrams of the beam-steering metasurface and coherent illumination. (a) A metasurface under the illumination of two coherent light beams at normal incidence. The output light forms a relatively narrow beam and the deflection angle $\theta$ is tuneable. (b) A unit cell of the metasurface.

The beam-steering metasurface is an array of silicon nanopillars on top of a glass substrate. Each unit cell consists of 10 equal-height, equal-spaced pillars with a gradient of diameter. The pillars are designed to support both electric and magnetic resonances close to the working wavelength of $555 \mathrm{~nm}$. The dimensions of these silicon nanopillars have been carefully chosen to achieve designed phase shift. For the electric resonance of the nanopillars, a large and nearly constant phase shift is observed across the whole unit cell of the metasurface. Meanwhile, for the magnetic resonance, the phase shift is significantly smaller. This contrast enables a phase gradient that responds to changes in the illumination condition.

\section{Characteristics of beam steering}

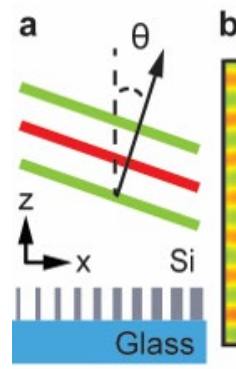

b
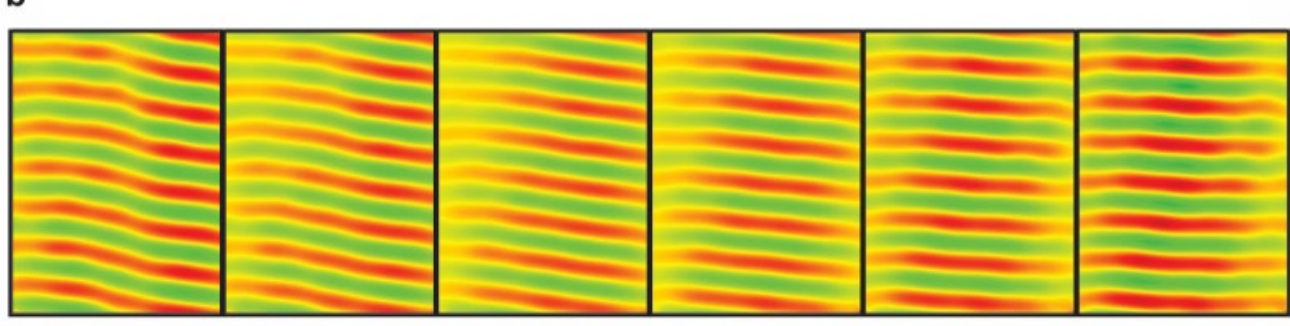

$E$

C
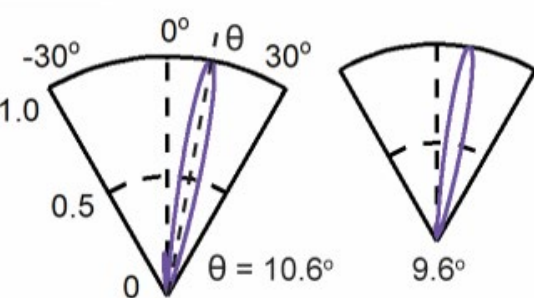

$9.6^{\circ}$

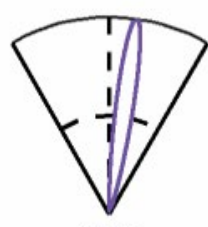

$8.1^{\circ}$

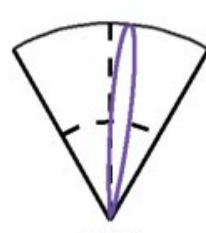

$5.7^{\circ}$

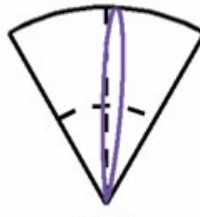

$2.9^{\circ}$

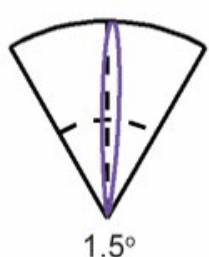

Fig. 2. Continuous beam steering of the metasurface. (a) Schematic diagram of the beam steering. (b) Electric field maps of the output light under six different illumination conditions. Each map covers an area that is 3 $\mu \mathrm{m}$ in width and between $1 \mu \mathrm{m}$ and $5 \mu \mathrm{m}$ above the metasurface. The field of all the maps is normalised against that of the free-space incident beam. (c) Corresponding radiation intensity patterns. All the patterns are normalised against the respective beam maximum. Only the first pattern is fully annotated due to space limitation.

Figure 2 shows light scattered by the beam-steering metasurface, which is illuminated by two $x$-polarised, counter-propagating incident beams at normal incidence. The first panel in Fig. $2 \mathrm{~b}$ is the output electric field with the metasurface at the electric antinode of the standing wave formed by the incident light. It shows a steering angle of $10.6^{\circ}$ (Fig. 2c), which is induced by the phase shift of the electric resonance of the nanopillars across a unit cell. As the phase shift of the magnetic resonance is significantly smaller, the steering angle is only $1.5^{\circ}$, as shown in the last panels in Figs. $2 \mathrm{~b}$ and $2 \mathrm{c}$. A continuous change in the steering angle is observed at the four intermediate states, corresponding to a gradual change in the response of the metasurface between the two extreme states.

We further show that rearranging the order of the nanopillars leads to a curved phase gradient that also responds to the coherent illumination. The curvature is large with the metalens at the electric antinode of the standing wave, due to the large difference in the phase shift of the electronic resonance between the pillars. The curvature decreases gradually as the illumination condition shifts over intermediate states towards the magnetic antinode. This change in the phase gradient leads to a change of $18 \%(\sim 20 \mu \mathrm{m})$ in the focal length.

\section{References}

[1] E. Plum et al. "Controlling the Optical Response of 2D Matter in Standing Waves," ACS Photonics 4, 3000-3011 (2017).

[2] N. Yu et al. "Light propagation with phase discontinuities: generalized laws of reflection and refraction," Science 334, 333-337 (2011). 